\title{
Angiogenesis modulation by exogenous antioxidants
}

\author{
DOROTA MAGDALENA RADOMSKA-LEŚNIEWSKA', BARBARA JOANNA BAEAN², \\ PIOTR SKOPIŃSKI
}

${ }^{1}$ Department of Histology and Embryology, Biostructure Centre, Medical University of Warsaw, Poland

${ }^{2}$ Department of Immunology Biochemistry and Nutrition, Medical University of Warsaw, Poland

\begin{abstract}
Co-operation of the endogenous and exogenous defense system maintains redox homeostasis and is essential for health. The endogenous defense system includes enzymatic (e.g. superoxide dismutase, catalase) and non-enzymatic, low molecular-weight scavengers (e.g. glutathione, ascorbic acid). Pathogenesis of many serious diseases (e.g. cancer, ischemic heart disease) includes oxidative stress which can disturb angiogenesis, the process of formation of new blood vessels sprouting from the existing one. Antioxidants, through reduction of oxidative stress and influence on neovascularization, may modulate progress and results of therapy in those diseases where such processes play an important role. Herein the impact of exogenous antioxidants on angiogenesis and factors modulating this process is presented. Most synthetic antioxidants whose activity has been described (namely $N$-acetylcysteine, pentoxifylline, synthetic analogue of curcumin, synthetic analogue of epigallocatechin-3 gallate [EGCG], tripertenoids) exert an inhibitory effect on neovascularization. A similar effect was also exhibited by several natural origin antioxidants (e.g. resveratrol, EGCG), which suggests that their application in therapy might normalize excessive angiogenesis. Some natural origin antioxidants e.g. purple coneflower and preparations consisting of natural antioxidants such as Padma 28 and Immunal forte increase a too low baseline level of angiogenesis and decreases a too high level. These preparations exert a regulatory effect on and may normalize neovascularization. They can be used in the case of diseases associated with too low or too high angiogenesis.
\end{abstract}

Key words: plant origin antioxidants, synthetic antioxidants, natural antioxidants, angiomodulatory properties, angiogenesis, reactive oxygen species.

(Centr Eur J Immunol 2017; 42 (4): 370-376)

\section{Introduction}

Pathogenesis of several serious human diseases is related to oxidative stress and pathological neovascularization. Oxidative stress, the imbalance between production of reactive oxygen species (ROS) and antioxidant mechanisms, disturbs redox homeostasis in a single cell and the organism [1]. It generates damage of cellular components, disturbances of their biological function and can lead to metabolism dysfunction [2]. A close relationship between ROS and neovascularization indicates that oxidative stress influences the angiogenesis process, too. Angiogenesis is the process of formation of new blood vessels sprouting from the existing one. Key mediators triggering formation of new vessels (e.g. vascular-epithelial growth factor (VEGF), fibroblast growth factor (FGF), matrix metalloproteinases (MMP), some interleukins (including IL-8), transforming growth factor $\alpha$ (TGF- $\alpha$ ), some adhesion molecules e.g. ICAM-1 (CD 54)), as well as hindering this process (e.g. tissue inhibitors of metalloproteinases (TIMP), interferons, TGF- $\beta$, IL-10, IL-4 and IL-12, IL-18) show at the same time a respective pro- or anti-angiogenic activity [3, 4].

In physiological conditions, angiogenesis influences the menstrual cycle, embryogenesis and wound healing, but its disturbances underlie many serious diseases, called angiogenesis-dependent diseases [2]. Excessive, pathological neovascularization has been reported e.g. in the case of neoplastic tumors, endometriosis, psoriasis, rheumatoid arthritis (RA), when its too low level is detected in e.g. neurodegenerative diseases, diabetes, hypertension, and ischemic heart disease. At the same time, all these diseases are accompanied by a chronic inflammatory state, and their pathomechanism is usually associated with oxidative stress [3, 4].

Antioxidants have been defined as substances that, when present at low concentrations, compared to oxidizable compound (e.g. DNA, lipids, proteins, carbohydrates), significantly delay or prevent substrate oxidation $[5,6]$. They neutralize free oxygen radicals, including ROS, and their activity, and counteract consequences of oxidative stress. ROS at high doses become deleterious, exhibiting

Correspondence: Dorota Magdalena Radomska-Leśniewska, Department of Histology and Embryology, Biostructure Centre,

Medical University of Warsaw, 5 Chalubinskiego St., 02-004 Warsaw, Poland, e-mail: dradomska@wum.edu.pl

Submitted: 30.10.2017; Accepted: 31.10.2017 
pathophysiological actions and as mentioned above, can lead to oxidative stress. On the contrary, low doses of ROS are beneficial for normal physiological actions.

The antioxidant defense system includes both endogenous and exogenous antioxidants. Antioxidant enzymes such superoxide dismutase (SOD), catalase (CAT), glutathione peroxidase (GPx), heme oxygenase and non-enzymatic, low molecular-weight scavengers (e.g. glutathione (GSH), uric acid, coenzyme Q, lipoic acid, ascorbic acid, tocopherol) belong to the endogenous defense system. The exogenous defense system includes antioxidants that can be grouped depending to their origin as natural (e.g. carotenoids and polyphenols, with the diet being the main source), nature identical (e.g. vitamin C, vitamin E) and synthetic - synthetized by the industry (e.g. N-acetylcysteine) [6]. The endogenous and exogenous defense system maintains or re-establishes redox homeostasis, such as during the regeneration of vitamin $\mathrm{E}$ by glutathione (GSH) or vitamin $\mathrm{C}$ to prevent lipid peroxidation processes, which can affect membrane fluidity and damage membrane proteins [7]. Through reduction of oxidative stress and the influence on neovascularization, antioxidants may modulate progress and results of therapy in those diseases, where such processes play an important role.

\section{Angiogenesis inhibition by synthetic antioxidants}

Several studies demonstrated that synthetic antioxidants were able to reduce or inhibit angiogenesis in both, in vitro or in vivo assays.

NAC belongs to synthetic antioxidants. Thanks to the presence of thiol groups, it directly neutralizes ROS. It also exerts indirect antioxidative and detoxifying activity, as glutathione precursor [5]. This drug has been long used in the clinical setting, especially in the treatment of pulmonary diseases (including chronic obstructive pulmonary disease (COPD), infections, cystic fibrosis) as a mucolytic agent, although the clinical effect of NAC may also result from its antioxidative and anti-inflammatory activity [2, 5]. NAC diminished transcription factor NF- $\kappa \mathrm{B}$ which regulates a lot of proinflammatory and antiapoptotic genes. It also inhibit c-jun N-terminal kinase (INK), p38 Erk, stress activated protein kinase (SAPK), as well as STAT and other redox-sensitive pathways. NAC exhibits also anti-inflammatory properties by downregulating inflammatory cytokines such as IL-8, IL-6 and tumor necrosis factor (TNF) $[2,5]$.

NAC presents also anti-angiogenic activity. The influence of a broad range of concentrations (0.01-30 mM) of $\mathrm{N}$-acetylcysteine (NAC) on the cells of endothelial line (ECV 304) and bronchial epithelial line (H292) in cell cultures was presented by us [8].

In this study NAC indirectly inhibited the inflammatory process and angiogenesis, through suppressing expres- sion of pro-inflammatory and pro-angiogenic molecules: ICAM-1 and IL-8, in activated endothelial and epithelial cells. NAC inhibited $(10 \mathrm{mM}$ and $30 \mathrm{mM})$ the level of soluble ICAM-1 as well.

It was also shown [9] that NAC inhibits spontaneous production of IL-8 (20-30 mM NAC) and MMP-9 (10-30 mM NAC), as well as expression of membrane-bound ICAM-1, through cells from bronchoalveolar lavage (BAL) obtained from sarcoidosis and idiopathic fibrosis (IPF) patients in vitro. While decreasing the expression of angiogenesis- and inflammatory state-stimulating factors, NAC indirectly inhibits these processes. This effect was dose-dependent. Total inhibition was seen at the level of $30 \mathrm{mM}$ NAC. This drug inhibited also spontaneous expression of ICAM-1 as evaluated with immunohistochemical methods in lymphocytes and macrophages originating from BAL from both patient groups. In all the described cases NAC at a concentration of $30 \mathrm{mM}$ caused total inhibition of CD 54 expression.

The investigations of Henry et al. [10] and Ishii et al. [11] also indicate that BAL from sarcoidosis and IPF patients shows elevated concentrations of pro-angiogenic, pro-inflammatory cytokines, such as IL-1, IL-2, IL-6, IL-8, MMP-9, and TNF. Recent reports demonstrate that in sarcoid granulomas mainly macrophages M2 accumulate, which in contrast to M1 show expression of a strong angiogenesis stimulator - VEGF. Thus, because of neovascularization disturbances and chronic inflammatory state in the pulmonary tissue, drugs normalizing these parameters in patients should bring positive therapeutic effects $[2,12]$.

Other investigators show angiogenesis and endothelial cells invasion downregulation by NAC through inhibition of MMP activity [13]. VEGF, the most potent stimulator of angiogenesis, was also diminished by this antioxidant in an in vivo study [14].

Inhibition of or at least decrease in excessive expression of ICAM- 1 and IL- 8 may contribute to a decrease in excessive angiogenesis and transmigration of leukocytes by vascular endothelium and limitation of the inflammatory state, or normalization of processes characteristic for the progression of many pulmonary diseases associated with inflammation and neovascularization disturbances.

Another synthetic antioxidant derived from natural polyphenol Curcumin (Curcuma longa) exhibits antioxidant, antiapoptotic and antiangiogenic properties. It inhibits VEGF and its receptor-2 (VEGFR-2), FGF2, MMPs expression, angiopoietin 2 and 1 signaling as well as suppresses NO concentration in vessels, thus prevents developing of metastasis. Owing to this wide range of bioactivities and lack of severe toxicity, Curcumin was used in clinical trials on cancer, inflammatory diseases, diabetes, neurodegenerative, and cardiovascular disease $[15,16]$.

Triterpenoids, synthetic antioxidants derived from oleanolic acid, are known as inhibitors of inflammation and angiogenesis. Particularly, CDDO (2-cyano-3,12-dioxoolea- 
na-1,9(11)-dien-28-oic acid and its methyl ester (CDDO-Me) were described to suppress $\mathrm{NO}$ and $\mathrm{COX}-2$ induction, $\mathrm{I} \kappa \mathrm{B} \alpha$ kinase and inhibit TNF- $\alpha$ 's ability to induce VEGF expression. In vivo and in vitro studies confirmed strong anti-angiogenic properties of CDDO-Me [17].

Green tea (Camellia sinensis) contains a rich variety of catechins, but the most abundant polyphenol is epigallocatechin-3 gallate (EGCG). It has been shown that EGCG and other catechins from green tea and black tea display anti-cancer and anti-angiogenic properties. Synthetic analogues of EGCG: pro-EGCG and (-)-EGCG diminished expression of MMP2 and MMP9 and inhibited activity of NFאB, ERK, PI3K/Akt signalling pathways. Taken together these synthetic EGCG analogues display anti-angiogenic properties [18].

Another group of substances present in herbs and vegetal foods including tea, coffee and chocolate are methyloxantines. Pentoxifylline (3,7-dimethyl-1-(5-oxohexyl) xanthine, PTX) is a synthetic methylated xanthine derivate. It is known as a competitive nonselective phosphodiesterase inhibitor which raises intracellular cAMP, activates phosphokinase A [19, 20] and leukotriene [21] synthesis, and reduces inflammation. PTX exhibits antioxidant and anti-tumor necrosis factor alpha (TNF- $\alpha$ ) properties. This drug is widely used in clinic particularly in patients suffering from peripheral vascular diseases e.g. as intermittent claudication of upper and lower limbs. The protective effects of PTX in pathogenesis of polycystic ovary syndrome have been recently reported [22]. Pathogenesis of this disease includes chronic low-grade inflammation and oxidative stress.

Bałan et al. [23] presented inhibition by PTX angiogenic activity of Sarcoma L-1 murine cell line in the cutaneous angiogenesis test. It was also demonstrated in this paper that PTX delayed appearance and reduced volume of Sarcoma L-1 tumors in recipient syngeneic Balb/c mice. PTX exerted synergistic inhibitory action with nonsteroidal anti-inflammatory drug, sulindac. Kamran and Gude presented an inhibitory effect of PTX (melanoma model) on angiogenesis, tumor growth and melanoma-derived metastatic tumor development in lungs. They indicated STAT3 signaling as a target of PTX inhibitory effect [24]. PTX was also reported to promote growth of murine colon adenocarcinoma and development of adenocarcinoma derived metastatic tumors in the liver and lungs. This effect, according to authors, has been explained by cytotoxic lymphocytes and inhibition by PTX perforin-dependent natural cytotoxicity in vitro [25-28].

Antiangiogenic activities were also described for synthetic antioxidants: Nomega-nitro-L-arginine methyl ester (L-NAME) and N5-(1-iminoethyl)-L-ornithine dihydrochloride. They decreased endothelial cell functions through down-regulation of the endothelial nitric oxide synthetase activity [29].

Most synthetic antioxidants, whose activity was herein described, exert an inhibiting effect on neovascularization, which suggests that their application in therapy might normalize excessive angiogenesis. The use of these antioxidants in the treatment of diseases associated with a too low angiogenesis level is risky as it may bring adverse results.

\section{Angiogenesis regulation by some natural antioxidants}

Majority of exogenous antioxidants are derived from plants, fungi, microorganisms or animals so they are called natural. Most available preparations with antioxidant properties belong to this group.

Purple coneflower (Echinacea purpurea) has proven anti-oxidative, anti-inflammatory and immunostimulating properties. It is used as an adjunctive treatment (in Germany it is registered as a drug) in diseases associated with immunity disturbances, particularly often in viral, bacterial and fungal infections of the respiratory tract [30].

An aqueous-alcoholic extract of purple coneflower in the form of tablets (IMMUNAL forte, Lek, Slovenia) administered to healthy volunteers, stimulated angiogenic activity in their mononuclear leukocytes (MNC) in the test of cutaneous angiogenesis (LIA) in mice [31]. IMMUNAL forte was administered to 6 healthy volunteers in the form of 1 tablet $(80 \mathrm{mg})$ and 8 healthy volunteers in the form of 2 tablets $(160 \mathrm{mg}$ ) daily for 7 days. The control group included healthy donors ( 8 people) receiving 1 placebo tablet daily. Angiogenic activity of peripheral blood leukocytes of evaluated persons before and after 7 days of treatment with purple coneflower was strongly stimulated by both evaluated doses of the preparation. These results constitute a confirmation of the previous studies on the preparation (IMMUNAL in the form of juice) with participation of healthy volunteers, which have proven an effect stimulating cellular immunity [32]. Moreover, E. purpurea extract was present to enhance immunity in mice [33].

An angiostimulatory effect of $E$. purpurea was achieved when this herb was administered to healthy volunteers' organism, with a physiological (normal) level of immune function and angiogenesis. As mentioned above, the excessive angiogenic response occurs in some diseases such as cancer, sarcoidosis and rheumatoid arthritis [2, 30]. E. purpurea is able to diminish abnormally high angiogenesis and restore it to the physiological level, so it acts as a regulator of the disturbed function. It was proved that E. purpurea extract lowered neovascular reaction induced in the mouse skin by human cancer cells. Besides angiogenic response induced in the mouse skin by syngeneic L1-sarcoma cells as well as VEGF concentration in L1 sarcoma tissue $[33,34]$ was also reduced by this herb demonstrate that the drug may normalize the angiogenesis process (it increases a too low baseline level of angiogenesis and decreases a too high level).

PADMA 28, preparation produced by the Swiss company, PADMA AG, consists of 20 natural ingredients 
(usually medicinal herbs) and originates from traditional Tibetan medicine. It is suggested that the preparation exhibits strong angioprotective, antioxidative, immunostimulating, anti-inflammatory, anti-peroxidative, cytoprotective and anabolic activity. According to the producer, chronic illnesses with inflammatory, atherosclerotic (including pathology of the peripheral and central vascular system) and degenerative background, as well as infectious diseases (in particular chronic, recurring infections of the respiratory tract) constitute the indication to use this supplement [35].

In our research [36], PADMA 28 was administered to mice per os, at a dose of $5.8 \mathrm{mg}$ and $0.085 \mathrm{mg} /$ day/mouse, in $40 \mu$ of water for 7 days, while animals from control groups received $40 \mu \mathrm{l}$ of water. Then, mice were bled under anesthesia, sacrificed, and spleens were harvested. Isolated splenocytes were implanted intradermally into recipient mice (Balb/c x C3H). After 3 days, new blood vessels induced by angiogenic factors released by implanted splenocytes (LIA test) were calculated. Studies presented in this paper prove that both doses of PADMA 28 preparation cause a statistically significant, dose-dependent increase in the number of new blood vessels. Moreover, the number of peripheral blood granulocytes and lymphocytes after 7 days of feeding with a lower dose of PADMA 28, increased significantly. At the same time, metabolic activity of peripheral blood granulocytes, determined by the intensity of their chemiluminescence, decreased after 7 days of feeding with a higher dose of preparation. This suggests that the lower dose, which also stimulated angiogenesis, may be safer in therapy. Results of this paper suggest that PADMA 28 preparation may be used in the case of diseases associated with disturbed angiogenesis, e.g. Alzheimer disease, diabetes, atherosclerosis, arterial hypertension, ischemic heart disease, and stroke [37]. Our earlier studies on PADMA 28 preparation demonstrate that the drug may normalize the angiogenesis process (it increases a too low baseline level of angiogenesis and decreases a too high level). Antiangiogenic activity of PADMA 28 has been demonstrated e.g. on human renal cancer cells [38]. Padma 28 was also reported to inhibit migration of cancer prostate cells [39]. Wang et al. [40] described antiangiogenic activity of this preparation by suppressing AKT/GSK-3 $\beta$ pathways in an in vitro and in vivo angiogenesis assay. Thus, this supplement may be used in the diseases with a high level of angiogenesis, too.

\section{Natural antioxidants inhibited angiogenesis}

A lot of other natural substances with antioxidant properties inhibiting angiogenesis have been described.

Echinacea purpurea is one of the components of Reumaherb preparation. Residual components are flowers of queen-of-the-meadow (Filipendula ulmaria) and the extract from grapple plant root (Harpagophytum procumbens).
Until now, Reumaherb was used mainly in the adjuvant treatment of inflammatory and degenerative diseases of joints, e.g. rheumatoid arthritis and pain syndromes of soft tissues of the locomotor system. The preparation shows anti-inflammatory, analgesic, anti-oxidative, immunomodulatory and regeneration-stimulating properties [30]. As our study indicates, Reumaherb can display anti-angiogenic properties when applied orally at a dose of $1.2 \mathrm{mg}$ per mice. In this research immunological angiogenesis induced by bronchoalveolar cells (BAL) isolated from patients with sarcoidosis and grafted into Balb/C mice skin was diminished [41] by this preparation. Similar experiments with Reumaherb was performed on mononuclear cells derived from RA patients. Reumaherb applied orally to the mice at daily doses of $1.2 \mathrm{mg}$ per mice diminished angiogenic response of mononuclear cells [30].

Resveratrol is a polyphenolic compound with antioxidative properties found in grapes, red wine and other fruits. Brakenhielm [42] demonstrated the inhibitory effect of resveratrol on angiogenesis and inflammation in both in vitro and in vivo models. Resveratrol reduced endothelial cells growth stimulated by FGF-2 and VEGF. It diminished in a dose-dependent manner angiogenesis in a chick chorioallantoic membrane assay [42]. Besides it inhibited corneal neovascularization induced by VEGF and FGF-2. Antiangiogenic properties of this substance rely on the influence of VEGF signaling. Resveratrol at a dose of 1-2.5 $\mu \mathrm{M}$ exposure of HUVEC cells blocked VEGF mediated migration and tube formation as well as tyrosine phosphorylation of vascular endothelial cadherin and $\beta$-catenin. Besides VEGF-induced endogenous Src kinase activation was strongly inhibited by 1 and $2.5 \mu \mathrm{M}$ of resveratrol $[42,43]$. Furthermore, resveratrol exerts antiangiogenic action by inhibiting NADPH oxidase (NOX-1) and monocyte chemotactic protein-1 production [44]. NOX are the main sources of ROS in endothelial cells. NOX regulates angiogenesis through production of VEGF and its receptor VEGR-2, MMPs and transcription factors that contribute to the angiogenesis process (e.g. NF-B, AP-1, STAT 1/3, E2F, CREB, HIF-1) [45, 46]. Seong reported that resveratrol suppresses VEGF secretion via inhibition of CXC-chemokine receptor 4 (CXCR4) expression in ARPE-19 cells [47]. A lot of studies concerned anticancer properties of resveratrol. It inhibited the growth of T241 fibrosarcoma in mice. In this study, resveratrol was administered orally at a concentration of $25 \mu \mathrm{M}$ [42]. It was reported that resveratrol and black tea polyphenol combination synergistically diminished mouse skin tumors growth by blocking of MAPKs and p53 [48]. Resveratrol and 5-fluorouracil inhibited cells growth and angiogenesis in B16 murine melanoma model [49]. Moreover, resveratrol inhibited oxidative stress based on DNA damages in lymphocytes of patients suffering from AMD, an eye disease caused by excessive pathological neovascularization in retina. Resveratrol at a concentration of 5-50 $\mu \mathrm{mlo} / 1$ 
reduced the DNA lesion in lymphocytes of patients with AMD as compared to healthy controls [50].

As mentioned above, consumption of drinking tea, rich in EGCG and other polyphenolic compounds, is the most popular beverage worldwide. Cao and Cao studied the angiogenic properties of EGCG. They show that EGCG inhibits proliferation of endothelial cells from bovine capillaries in a dose-dependent manner. Angiogenesis was also inhibited by EGCG in chick chorioallantoic membrane assay [51]. Besides green tea consumption (EGCG in a concentration similar to levels after drinking 2-3 cups of tea) inhibited angiogenesis in a model of corneal neovascularization in mice stimulated by VEGF [51]. It was also demonstrated that EGCG diminish angiogenesis through suppression of IL-8 production by human microvascular endothelial cells [52]. Inflammatory angiogenesis was also reduced by EGCG in an in vivo study [53]. Angiogenesis inhibition by EGCG was related to downregulation of MMP activity [54], removing ROS [55] and decreasing VEGF induction in human colon carcinoma [56]. A study of Junk et al. demonstrated that treatment with EGCG (1.5 mg/day/mouse for 20 days) athymic BALB/C nude mice inoculated subcutaneously with human colon cancer cells diminished the number of tumor microvessels, cell proliferation and increase in apoptosis of tumoral and endothelial cells [56]. EGCG inhibited transcription factor AP-1, binding to DNA. AP-1 upregulates MMP-1 gene and plays an important role in metastatic behavior of cancer cells [57].

\section{Conclusions}

Disturbances of angiogenesis might have serious medical consequences. An abnormally high angiogenesis level is characteristic for cancer, one of the most dangerous and still incurable diseases. In turn, too low angiogenesis can be observed in diabetes mellitus or ischemic heart disease. Pathogenesis of these and many others civilization diseases include also oxidative stress. Co-operation of the endogenous and exogenous defense system maintain redox homeostasis and is essential for health. In this review, the influence of exogenous antioxidants on various parameters of neovascularization was described. Most presented synthetic antioxidants exert an inhibitory effect on neovascularization. A similar effect is exhibited also by some natural origin antioxidants (e.g. resveratrol, EGCG), which suggests that their application in therapy might normalize excessive angiogenesis. Although, the use of these antioxidants in the treatment of diseases associated with a too low angiogenesis level is risky as it may bring adverse results. Some natural origin antioxidants e.g. purple coneflower and preparation consisting natural antioxidants such Padma 28 increase a too low baseline level of angiogenesis and decreases a too high level. These preparations exert a regulatory effect on angiogenesis and their action may normalize the disturbed angiogenesis process. These natu- ral origin antioxidants can be used in the case of diseases associated with too low or too high angiogenesis.

The authors declare no conflict of interest.

\section{References}

1. Devasagayam TP, Tilak JC, Boloor KK, et al. (2004): Free radicals and antioxidant in human health: current status and future prospects. JAPI 52: 794-804.

2. Radomska-Leśniewska DM, Hevelke A, Skopiński P, et al. (2016): Reactive oxygen species and synthetic antioxidants as angiogenesis modulators. Pharmacol Rep 68: 462-471.

3. Carmeliet P, Jain RK (2011): Molecular mechanisms and clinical applications of angiogenesis. Nature 473: 298-307.

4. Brambilla D, Mancuso C, Scuderi MR, et al. (2008): The role of antioxidant supplement in immune system, neoplastic, and neurodegenerative disorders: a point of view for an assessment of the risk/ benefit profile. Nutr J 30: 7-29.

5. Sadowska AM, Manuel-Y-Keenoy B, De Backer WA (2007): Antioxidant and anti-inflammatory efficacy of NAC in the treatment of COPD: discordant in vitro and in vivo dose-effects: a review. Pulm Pharmacol Ther 20: 9-22.

6. Halliwell B (1999): Antioxidant defence mechanisms: from the beginning to the end (of the beginning). Free Radic Res 31: 261-272.

7. Valko M, Leibfritz D, Moncol J, et al. (2007): Free radicals and antioxidants in normal physiological functions and human disease. Int J Biochem Cell Biol 39: 44-84.

8. Radomska-Leśniewska DM, Skopińska-Różewska E, Jankowska-Steifer E, et al. (2010): N-acetylcysteine inhibits IL-8 and MMP-9 release and ICAM-1 expression by bronchoalveolar cells from interstitial lung diseases patients. Pharmacol Rep 2010; 62: 131-138.

9. Radomska-Leśniewska DM, Sadowska AM, van Overveld F, et al. (2006): Influence of $\mathrm{N}$-acetylcysteine on ICAM-1 expression and IL-8 release from endothelial and epithelial cells. J Physiol Pharmacol 57 Suppl 4: 325-334.

10. Henry MT, McMahon K, Mackarel AJ, et al. (2002): Matrix metalloproteinases and tissue inhibitor of metalloproteinase-1 in sarkoidosis and IPF. Eur Resp J 20: 1220-1227.

11. Ishii Y, Kitamura S (1995): Elevated level of soluble ICAM in serum and BAL fluid in patient with active sarcoidosis. Chest 107: 1636-1640.

12. Martines FO, Gordon S (2014): The M1 and M2 paradigm of macrophage activation: time for rassessment. F1000 Prime Repp 6: 13.

13. Zafarullah M, Li WQ, Sylvester J, Ahmad M (2003): Molecular mechanisms of $\mathrm{N}$-acetylcysteine actions. Cell Mol Life Sci 60: 6-20.

14. Gao P, Zhang G, Dinavahi R, et al. (2007): HIF-dependent anti-tumorigenic effect of anti-oxidants in vivo. Cancer Cell 12: 230-238.

15. Fan X, Zhang C, Liu DB, et al. (2013): The clinical applications of curcumin: current state and the future. Curr Pharm Des 19: 2011-2031.

16. Prauchner CA (2014): Angiogenesis inhibition by antioxidants. Internat J Biomed Science and Engineer 2: 7-19.

17. Wang YY, Zhe H, Zhao R, et al. (2014): Preclinical evidences toward the use of triterpenoid CDDO-Me for solid cancer prevention and treatment. Mol Cancer 13: 30. 
18. Wang CC, Xu H, Man GC, et al. (2013): Prodrug of green tea epigallocatechin-3-gallate (Pro-EGCG) as a potent antiangiogenesis agent for endometriosis in mice. Angiogenesis 16: 59-69.

19. Deree J, Martins JO, Melbostad H, et al. (2008): Insights into the regulation of TNF-alpha production in human mononuclear cells: the effects of non-specific phosphodiesterase inhibition. Clinics 63: 321-328.

20. Marques LJ, Zheng L, Poulakis N, Guzman J, Costabel U (1999): Pentoxifylline inhibits TNF-alpha production from human alveolar macrophages. Am J Respir Crit Care Med 159: 508-511.

21. Peters-Golden M, Canetti C, Mancuso P, Coffey MJ (2005): Leukotrienes: underappreciated mediators of innate immune responses. J Immunol 174: 589-594.

22. Rezvanfar MA, Saadat S, Shojaei Saadi HA, et al. (2015): Cellular and molecular mechanisms of pentoxifylline's beneficial effects in experimental polycystic ovary. Theriogenol 83: 968-977.

23. Bałan BJ, Demkow U, Skopiński P, et al. (2017): The effect of pentoxifylline on L-1 sarcoma tumor growth and angiogenesis in Balb/c mice. Cent Eur J Immunol 42: 131-139.

24. Kamran MZ, Gude ROP (2013): Pentoxifylline inhibits melanoma tumor growth and angiogenesis by targeting STAT3 signaling pathway. Biomed Pharmacother 67: 399-405.

25. Lazarczyk M, Grzela T, Niderla J, et al. (2004): Differential influence of pentoxifylline on murine colon adenocarcinoma- and melanoma-derived metastatic tumor development in lungs. Oncol Rep 11: 1121-1125.

26. Grzela T, Lazarczyk M, Niderla J, et al. (2003): Pentoxifylline promotes development of murine colon adenocarcinoma-derived metastatic tumors in liver. Oncol Rep 10: 1805-1809.

27. Lazarczyk M, Grzela T, Niderla J, et al. (2003): Pentoxifylline inhibits leukocyte infiltration and splenocyte cytotoxicity against murine colon adenocarcinoma. Oncol Rep 10: 839-843.

28. Lazarczyk M, Grzela T, Korczak-Kowalska G, et al. (2002): Pentoxifylline inhibits perforin-dependent natural cytotoxicity in vitro. Oncol Rep 9: 423-426.

29. Polytarchou C, Papadimitriou E (2005): Anioxidants inhibit human endothelial cell functions through down-regulation of the endothelial nitric oxide synthetase activity. Eur J Pharmacol 510: 31-38.

30. Radomska-Leśniewska DM, Skopiński P, Bałan BJ, et al. (2015): Angiomodulatory properties of Rhodiola spp. and other natural antioxidants. Centr Eur J Immunol 40: 249-262.

31. Skopińska-Różewska E, Sommer E, Bałan B, et al. (2010): The in vivo effect of dry hydro-alcoholic extract of Echinacea purpurea on angiogenic activity of human blood mononuclear cells. Centr Eur J Immunol 35: 223-226.

32. Rogala E, Skopińska-Różewska E, Wasiutyński A, et al. (2008): Echinacea purpurea diminishes neovascular reaction induced in mice skin by human cancer cells and stimulates non-specific cellular immunity in humans. Centr Eur J Immunol 33: 127-130.

33. Bany J, Siwicki AK, Zdanowska D, et al. (2003): Echinacea purpurea stimulates cellular immunity and anti-bacterial defence independently of the strain of mice. Pol J Vet Sci 6 (Suppl 3): 3-5.

34. Wasiutyński A, Bałan B, Skopińska-Różewska E, et al. (2009): The effect of Echinacea purpurea on the morphology, angiogenic activity and vascular endothelial growth factor (VEGF) concentration of murine L-1 sarcoma tumors. Centr Eur J Immunol 34: 38-41.
35. Skopiński P, Radomska-Leśniewska DM, Sokolnicka I, et al. (2013): In vivo stimulatory effect of multi-component herbal remedy PADMA 28 on mitogen-induced proliferation of mice splenic lymphocytes and their chemokinetic activity. Pol J Vet Sci 4: 701.

36. Radomska-Leśniewska DM, Skopiński P, Niemcewicz M, et al. (2013): The effect of anti-inflammatory and antimicrobial herbal remedy PADMA 28 on immunological angiogenesis and granulocytes activity in mice. Mediators of Inflammation 2013: 853475.

37. Ginsburg I, Rozenstein-Tsalkovich L, Koren E, Rosenmann H (2011): The herbal preparation Padma 28 protects against neurotoxicity in PC12 cells. Phytother Res 25: 740-748.

38. Skopińska-Różewska E, Pastewka K, Wasiutyński A, et al. (2010): The influence of herbal remedies on cutaneous angiogenesis induced in mice after grafting of human kidney cancer tissue. Centr Eur J Immunol 35: 199-203.

39. Kim EJ, Hong JE, Lim SS, et al. (2012): The hexane extract of Saussurea lappa and its active principle, dehydrocostus lactone, inhibit prostate cancer cell migration. J Med Food 15: 24-32.

40. Wang CY, Tsai AC, Peng CY, et al. (2012): Dehydrocostuslactone suppresses angiogenesis in vitro and in vivo through inhibition of AKT/GSK-3beta and mtor signaling pathways. PLoS One 7: e31195.

41. Radomska-Leśniewska DM, Skopińska-Różewska E, Demkow U, et al. (2016): Natural herbal remedy modulates angiogenic activity of bronchoalveolar lavage cells from sarcoidosis patients. Centr Eur J Immunol 41: 25-30.

42. Bríkenhielm E, Cao R, Cao Y (2001): Suppression of angiogenesis, tumor growth, and wound healing by resveratrol, a natural compound in red wine and grapes. FASEB J 15: 1798-1800.

43. Prauchner CA (2014): Angiogenesis inhibition by antioxidants. Intern J Biomed Scien Engineer 2: 7-19.

44. Park DW, Baek K, Kim JR, et al. (2009): Resveratrol inhibits foam cell formation via NADPH oxidase 1-mediated reactive oxygen species and monocyte chemotactic protein-1. Exp Mol Med 41: 171-179.

45. Ushio-Fukai M, Tang Y, Fukai T, et al. (2002): Novel role of gp91phox-containing NAD(P)H oxidase in vascular endothelial growth factor-induced signaling and angiogenesis. Circ Res 91: 1160-1167.

46. Diebold I, Petry A, Hess J, Görlach A (2010): The NADPH oxidase subunit NOX4 is a new target gene of the hypoxia-inducible factor-1. Mol Biol Cell 10: 2087-2096.

47. Seong H, Ryu J, Jeong JY, et al. (2015): Resveratrol suppresses VEGF secretion via inhibition of CXC-chemokine receptor 4 expression in ARPE-19 cells. Mol Med Rep 12: 1479-1484.

48. George J, Singh M, Srivastava AK, et al. (2011): Resveratrol and black tea polyphenol combination synergistically suppress mouse skin tumors growth by inhibition of activated MAPKs and p53. PLoS One 6: e23395.

49. Lee SH, Koo BS, Park SY, Kim YM (2015): Anti-angiogenic effects of resveratrol in combination with 5-fluorouracil on B16 murine melanoma cells. Mol Med Rep 12: 2777-2783.

50. Szaflik JP, Stańczyk M, Skłodowska A, et al. (2013): Resveratrol may reduce oxidative stress in AMD patients. SOE 8-11 June 2013. Copenhagen, Denmark; Poster EP_RET-739.

51. Cao Y, Cao R (1999): Angiogenesis inhibited by drinking tea. Nature 398: 381.

52. Yang CS, Chen L, Lee MJ, et al. (1998): Blood and urine levels of tea catechins after ingestion of different amounts of 
green tea by human volunteers. Cancer Epidemiol Biomark Prev 7: 351-354.

53. Dona M, Dell'Aica I, Calabrese F, et al. (2003): Neutrophil restraint by green tea: inhibition of inflammation, associated angiogenesis, and pulmonary fibrosis. J Immunol 170: 43354341.

54. Maeda-Yamamoto M, Kawahara H, Tahara N, et al. (1999): Effects of tea polyphenols on the invasion on the matrix metalloproteinases acitivities of human fibrosarcoma HT1080 cells. J Agric Food Chem 47: 2350-2354.

55. Zhang G, Miura Y, Yagasaki K (2000): Supression of adhesion through antioxidative activity and invasion of hepatoma cell in culture by tea compounds adhesion. Cancer Lett 159: 169-173.

56. Jung YD, Kim MS, Shin BA, et al. (2001): EGCG, a major component of green tea, inhibits tumour growth by inhibiting VEGF induction in human colon carcinoma cells. Br J Cancer 84: 844-850.

57. Dong Z, Ma WY, Huang C, Yang CS (1997): Inhibition of tumour promoter-induced activator protein 1 activation and cell transformation by tea polyphenols, (-)-epigallocatechin gallate, and theaflavins. Cancer Res 57: 4414-4419. 